VOL. 53 (1996) [229-233]

\title{
A CONTINUOUS ANALOGUE AND AN EXTENSION OF RADÓ'S FORMULA
}

\author{
C.E.M. Pearce and J. PeČarić
}

A continuous analogue is derived for Radó's comparison formulæ. The analogue is then employed to provide a result which continues Radó's result and interpolates an inequality of Pittenger.

\section{INTRODUCTION}

Sándor [9] has recently proved the following result.

THEOREM A. If $f:[a, b] \rightarrow \mathbf{R}$ is positive, continuous and convex (respectively concave), then

$$
\frac{1}{b-a} \int_{a}^{b} f^{2}(x) d x \leqslant(\geqslant) \frac{1}{3}\left[f^{2}(a)+f(a) f(b)+f^{2}(b)\right],
$$

with equality only when $f$ is a linear function.

As special cases of this striking theorem he obtained such inequalities as

$$
3 / L(a, b)<1 / G(a, b)+2 / H(a, b)
$$

and

$$
I^{3}(a, b)>G^{2}(a, b) \exp [G(\ln a, \ln b)],
$$

where $L, G, H$ and $I$ denote respectively the logarithmic, geometric, harmonic and identric means of two given numbers $a, b$ both exceeding unity.

Sándor's result appears at first a somewhat isolated one. Its role in the canon of integral inequalities becomes more recognisable if it is recast in terms of extended logarithmic means and integral power means. This we do in Section 2. The form then assumed by (1) suggests a natural generalisation, which we establish.

The generalisation found in Section 2 provides a continuous analogue of a remarkable discrete inequality established by Radó [8] sixty years ago, special cases of which have been rediscovered repeatedly in the subsequent literature. In Section 3 we recapitulate Radó's result and show how a result of Pittenger [7] may be deduced from it as a special case. Finally, in Section 4, we piece together Pittenger's result, Radó's formulæ and our theorem from Section 2 together to provide a result which continues the Radó inequality and provides an interpolation of the Pittenger result.

Received 27th April, 1995.

Copyright Clearance Centre, Inc. Serial-fee code: 0004-9729/96 \$A.2.00+0.00. 
2. EXTENDED LOGARITHMIC AND INTEGRAL POWER MEANS

First recall the notion of the extended logarithmic means $L_{p}(a, b)$ of two positive numbers $a, b$. For $a \neq b$ these are defined by

$$
\begin{aligned}
L_{p}(a, b) & =\left[\frac{b^{p+1}-a^{p+1}}{(p+1)(b-a)}\right]^{1 / p}, \quad p \neq-1,0, \\
L_{-1}(a, b) & =L(a, b)=\frac{b-a}{\ln b-\ln a} \\
L_{0}(a, b) & =I(a, b)=\frac{1}{e}\left(\frac{b^{b}}{a^{a}}\right)^{1 /(b-a)}
\end{aligned}
$$

and for $a=b$ by

$$
L_{p}(a, a)=a .
$$

We shall need also the integral power means $M_{p}$ of a positive function $f$ on $[a, b]$, defined by

$$
M_{p}(f)= \begin{cases}{\left[\frac{1}{b-a} \int_{a}^{b} f(t)^{p} d t\right]^{1 / p},} & p \neq 0, \\ \exp \left[\frac{1}{(b-a)} \int_{a}^{b} \ln f(t) d t\right], & p=0 .\end{cases}
$$

In terms of these, (1) can be rewritten conveniently as

$$
M_{2}(f) \leqslant(\geqslant) L_{2}(f(a), f(b))
$$

This suggests the following generalisation of Theorem A.

THEOREM 1. If $f:[a, b] \rightarrow \mathbf{R}$ is positive, continuous and convex (respectively concave), then

$$
M_{p}(f) \leqslant(\geqslant) L_{p}(f(a), f(b))
$$

with equality only when $f$ is a linear function.

Proof: Denote by $K$ the linear function on $[a, b]$ given by $K(a)=f(a), K(b)=$ $f(b)$, that is,

$$
K(t)=\frac{t-a}{b-a} f(b)+\frac{b-t}{b-a} f(a), \quad t \in[a, b] .
$$

First suppose $f$ is convex. Then $f(t) \leqslant K(t)$ for $a \leqslant t \leqslant b$ and therefore

$$
M_{p}(f) \leqslant M_{p}(K) .
$$


Moreover, from the substitution

$$
x=\frac{t-a}{b-a} f(b)+\frac{b-t}{b-a} f(a)
$$

the definition of $M_{p}(K)$ gives immediately

$$
M_{p}(K)=L_{p}(f(a), f(b)),
$$

so that (3) gives (2) for the case of convexity. Further, $f(t)<K(t)$ for some $t \in(a, b)$ unless $f$ is linear, from which we derive the statement about equality for the convex case.

For $f$ concave we have with the same argument a reverse inequality in (3) and so also in (2). The statement concerning equality is derived similarly.

\section{RADÓ'S INEQUalities}

Theorem 1 above provides an analogue of a comparison given by Radó [8] for the integral power mean and the classical power mean of two positive numbers $x, y$, where the latter mean is defined by

$$
M_{p}(x, y)= \begin{cases}\left(\frac{x^{p}+y^{p}}{2}\right)^{1 / p}, & p \neq 0 \\ \sqrt{ }(x y), & p=0 .\end{cases}
$$

Radó's result may be cast as follows.

THEOREM B. Suppose $a, b, r$, are real numbers with $a<b$ and $f \in C[a, b]$ is a positive function. If $f$ is convex, then

$$
M_{r}(f) \geqslant M_{r_{1}}(f(a), f(b))
$$

whilst for $f$ concave

$$
M_{r}(f) \leqslant M_{r_{3}}(f(a), f(b))
$$

Here

$$
r_{1}= \begin{cases}\min \left(\frac{r+2}{3}, \frac{r \ln 2}{\ln (r+1)}\right), & r>-1, r \neq 0, \\ \min (2 / 3, \ln 2), & r=0, \\ \min ((r+2) / 3,0), & r \leqslant-1,\end{cases}
$$

and $r_{2}$ is defined by the same formula with min replaced by max. The values $r_{1}, r_{2}$ are best possible in the sense that the former inequality will fail for some choices of $f$ 
if $r_{1}$ is replaced by a larger number and the latter will fail for some choices of $f$ if $r_{2}$ is replaced by a smaller number.

The analysis in [8] used to derive this result is fairly detailed and hangs on comparison of each function concerned with a linear function. One consequence of this is that, for given values of $f(a)$ and $f(b)$, the 'best possible' constraint on the values of $r_{1}$ and $r_{2}$ is realised by a linear function.

From Theorem B we may deduce the following result, established by Pittenger [7] in 1980 .

THEOREM C. Suppose $a, b, r$ are real numbers with $0<a \leqslant b$. Then

$$
M_{r_{2}}(a, b) \geqslant L_{r}(a, b) \geqslant M_{r_{1}}(a, b) .
$$

The inequalities are tight. Equality of the leftmost and rightmost terms can arise if and only if $a=b$ or $r=1,-1 / 2$ or -2 .

Proof: Equality throughout (6) is immediate if $a=b$, so without loss of generality suppose $a<b$. Relations (4) and (5) both hold for the choice $f(x)=x$ in Theorem $B$ and reduce respectively to

$$
\begin{aligned}
& L_{r}(a, b) \geqslant M_{r_{1}}(a, b), \\
& L_{r}(a, b) \leqslant M_{r_{2}}(a, b) .
\end{aligned}
$$

Relation (6) follows at once. That (6) is tight follows from our observation about linearity and the choices of $r_{1}, r_{2}$ being best possible. The statement concerning equality for $a<b$ follows from the fact that, for $x, y$ distinct, $M_{p}(x, y)$ is strictly increasing in $p$ (see Kazarinoff $\left[4\right.$, p.64]), so that equality arises only when $r_{1}=r_{2}$.

REMARK. Many mathematicians have proved special cases of (6). Thus Lin [5] in 1979 proved

$$
G(a, b) \leqslant L(a, b) \leqslant M_{1 / 3}(a, b)
$$

(which is (6) for $r=-1$ ), Alzer [1] in 1985 proved

$$
M_{2 / 3}(a, b) \leqslant I(a, b) \leqslant M_{\ln _{2}}(a, b)
$$

(which is (6) for $r=0$ ), while Stolarsky [10] in 1980 proved that if $-2<r<-1 / 2$ or $r>1$ then

$$
L_{r}(a, b) \leqslant M_{(r+2) / 3}(a, b) .
$$

A probabilistic proof of (6) has been given by Székely [11]. Székely [12] considered this type of inequality for defining the "distance between means". For a proof of Theorem B see Lupaş and Lupaş [6] and for further extensions see Hartman [2].

Theorems $\mathrm{B}$ and $\mathrm{C}$ and our Theorem 1 may be melded together to continue the Radó result and give an interpolation of Pittenger's result. 
Theorem 2. Suppose $a, b, r$ are real numbers with $a<b$ and $f \in C[a, b]$ is strictly positive. If $f$ is convex, then

$$
M_{r_{3}}(f(a), f(b)) \geqslant L_{r}(f(a), f(b)) \geqslant M_{r}(f) \geqslant M_{r_{1}}(f(a), f(b)),
$$

whilst for $f$ concave

$$
M_{r_{1}}(f(a), f(b)) \leqslant L_{r}(f(a), f(b)) \leqslant M_{r}(f) \leqslant M_{r_{2}}(f(a), f(b)) .
$$

PROOF: In each case the consecutive inequalities are given respectively by Theorems $\mathrm{C}, 1$ and $\mathrm{B}$.

REMARK. For another type of interpolation of a special case of Theorem C see Imoru [3].

\section{REFERENCES}

[1] H. Alzer, 'Ungleichungen für $(e / a)^{a}(b / e)^{b}$ ', Elem. Math. 40 (1985), 120-123.

[2] P. Hartman, 'Convex functions and mean value inequalities', Duke Math. J. 39 (1972), 351-360.

[3] C.O. Imoru, 'The power mean and the logarithmic mean', Internat. J. Math. Math. Sci. 5 (1982), 337-343.

[4] N.D. Kazarinoff, Analytic inequalities (Holt, New York, 1961).

[5] T.P. Lin, 'The power mean and the logarithmic mean', Amer. Math. Monthly 81 (1979), 879-883.

[6] A. Lupaş and L. Lupaş, 'A class of inequalities for convex functions', Rev. Mat. elev. Timişoara 17 (1986), 11-23.

[7] A.O. Pittenger, 'Inequalities between arithmetic and logarithmic means', Univ. Beograd. Publ. Elektrotehn. Fak. Ser. Mat. 678-715 (1980), 15-18.

[8] T. Radó, 'On convex functions', Trans. Amer. Math. Soc. 37 (1935), 266-285.

[9] J. Sándor, 'On the identric and logarithmic means', Aeqationes Math. 40 (1990), 261-270.

[10] K.B. Stolarsky, 'The power and generalized logarithmic mean', Amer. Math. Monthly 87 (1980), 545-548.

[11] J.G. Székely, 'A probabilistic proof of inequalities of some means', Periodica Polytech. Chem. Eng. 16 (1979), 99-101.

[12] J.G. Székely, 'A classification of means', Ann. Univ. Sci. Budapest Eötvös Sect. Math. 18 $(1975 / 6), 129-133$.

Applied Mathematics Department

The University of Adelaide

Adelaide, SA 5005

Australia
Faculty of Textile Technology

University of Zagreb

Zagreb

Croatia 